\title{
Multi-aircraft conflict detection and resolution based on probabilistic reach sets
}

\author{
Yang Yang*, Jun Zhang*, Kai-quan Cai*, and Maria Prandini ${ }^{\dagger}$
}

\begin{abstract}
In this paper, a novel scheme to multi-aircraft conflict detection and resolution is introduced. A key feature of the proposed scheme is that uncertainty affecting the aircraft future positions along some look-ahead prediction horizon is accounted for via a probabilistic reachability analysis approach. In particular, ellipsoidal probabilistic reach sets are determined by formulating a chance-constrained optimization problem and solving it via a simulation-based method called scenario approach. Conflict detection is then performed by verifying if the ellipsoidal reach sets of different aircraft intersect. If a conflict is detected, then, the aircraft flight plans are redesigned by solving a second order cone program resting on the approximation of the ellipsoidal reach sets with spheres with constant radius along the look-ahead horizon. A bisection procedure allows to determine the minimum radius such that the ellipsoidal reach sets of different aircraft along the corresponding new flight plans do not intersect. Some numerical examples are presented to show the efficacy of the proposed scheme.
\end{abstract}

Index Terms-Air traffic control, Probabilistic reachability, Multi-aircraft Conflict Detection and Resolution, Randomized algorithms, Stochastic/uncertain systems.

\section{INTRODUCTION}

In this paper, we address multi-aircraft Conflict Detection and Resolution (CD\&R) on the mid-term time scale that characterizes the operations at the level of the Air Traffic Controllers (ATCs) in charge of guaranteeing safety in air travel. The idea is to provide ATCs with automatic support tools so as to simplify their task and, hence, enable them to handle safely higher traffic levels [20]. Each aircraft is assigned some flight plan to track, which consists of a sequence of timed way-points. In the current practice, a conflict is detected when the predicted distance between at least two aircraft gets smaller than a certain safety distance, and this distance is computed based on the nominal trajectories as determined by the aircraft flight plans. However, due to the uncertainty affecting the aircraft motion and, in particular, due to wind, the aircraft actual trajectories differ from the nominal trajectories, see e.g. [10], [29], [17], [9], [25]. This

This work was performed while the first author was visiting M. Prandini at the Politecnico di Milano, Italy. It is partly supported by the European Commission under the UnCoVerCPS project, grant number 643921, and by the National Basic Research Program of China (No. 2011CB707004), National Key Technologies R\&D Program of China (No. 2012BAG04B01), and China Scholarship Council.

*Y. Yang, J. Zhang, K. Cai are with School of Electronic and Information Engineering, Beihang University, XueYuan Road No. 37, 100191 Beijing, China $\{$ yangyang, caikq\} @ee.buaa.edu.cn, buaazhang jun@vip. sina. com

$\dagger$ M. Prandini is with Dipartimento di Elettronica, Informazione e Bioingegneria, Politecnico di Milano, via Ponzio 34/5, 20133 Milano, Italy maria.prandiniepolimi.it is particularly critical when aircraft are densely packed in the airspace. To account for uncertainty and avoid situations where a multi-aircraft encounter is predicted to be safe, while it is not, we introduce a different notion of conflict, which is based on the concept of probabilistic reach sets as explained next.

There is indeed a vast literature on reachability analysis, including the study of tools for reach sets computation, for various classes of systems, mainly deterministic, [3], [16], [13], [2], [21], [23]. Different representations of reach sets (polyhedra, zonotopes, ellipses) are adopted. In our context, the main source of uncertainty is wind, which is naturally described through a stochastic model. This calls for the notion of probabilistic reach set [12]. A probabilistic reach set is associated to a certain probability level $1-\epsilon, \epsilon \in(0,1)$, and includes all states that the system can reach except for a set of probability smaller than or equal to $\epsilon$. As $\epsilon$ approaches 0 , the standard notion of reach set comprising all reachable states is recovered.

Ellipsoidal uncertainty sets are commonly used in aeronautics [10], [18] to describe the uncertain aircraft position with respect to some reference trajectory: the tracking error is supposed to be Gaussian and uncorrelated in time, and the ellipses where the aircraft is confined along the prediction horizon are derived as level sets of independent Gaussian distributions. In this work, we adopt a method for reach sets computation that do not require any assumption on the tracking error distribution and correlation structure. Still we use ellipses to represent reach sets, since they are easily parameterized through their center and shape matrix, and their computation can be reduced to a convex optimization program. Following [12], probabilistic reach set are computed by formulating a chance-constrained optimization program, which is then solved via the so-called scenario approach [5], [6], [8]. This involves running a number of simulations of the aircraft trajectories that is inversely proportional to $\epsilon$. An air traffic simulator including a stochastic wind field model is adopted to this purpose [14]. Based on the probabilistic reach sets, a conflict can be defined as the event when reach sets of different aircraft intersect at some time instant. When a conflict is detected, then, the aircraft flight plans are re-designed so as to minimize the traveled distance while maintaining reach sets separated. This is achieved via a two-step approach. In the first step, ellipsoidal reach sets are replaced by spheres of the same constant radius $r$ along the look-ahead time horizon, and two-legged maneuvers are designed so as to keep the aircraft nominal trajectories at a distance larger than or equal to $r$. In the second step, the actual ellipsoidal reach sets are checked for intersection. Radius $r$ is progressively increased till no 
intersection occurs between the actual ellipsoidal reach sets. $\epsilon$ is eventually increased if the airspace is densely packed. Indeed, as $\epsilon$ grows to 1 , reach sets get smaller and smaller. It is then easier to solve conflicts but at the price of decreasing safety guarantees.

Note that this paper extends in a non trivial way our preliminary work in [33], by refining probabilistic reach set computation, and, more importantly, by proposing a completely different resolution strategy from that in [33], which is restricted to the two-aircraft case and rests on the solution to a non convex optimization problem. Results are presented with reference to the level flight case. Generalization of the approach to the more general 3 Dimensional (3D) case is straightforward but involves using a more complex simulation model. Compared with other probabilistic reachability-based approaches to CD\&R, our method is computationally more effective. As a matter of fact, those numerical methods resting on state space gridding and Markov chain approximation scale badly with the number of aircraft and with the dimension of the considered airspace region [17], [28], whereas those resorting to the standard Monte Carlo approach for estimating the probability of conflict up to an accuracy $\epsilon$ need a number of simulations that is inversely proportional to $\epsilon^{2}$ [29]. Alternative approaches based on an analytic approximation of the probabilistic reach sets are computationally attractive but they rest on simplifying assumptions on the aircraft dynamics and stochastic wind, which make the model not realistic, [29].

The rest of the paper unfolds as follows. In Section II we briefly describe the adopted model of the aircraft dynamics. We then present the chance-constrained approach to reach set computations and its randomized version in Section III. The two-step multi-aircraft CD\&R scheme is presented in Section IV. Section V shows some promising numerical results. Finally, some concluding remarks are given in Section VI.

\section{Model OF THE AIRCRAFT DYNAMICS}

The point mass model of the aircraft dynamics that we adopt for reach set computations was proposed in [14] and implemented in an air traffic simulator.

The model comprises three main components: a continuous dynamics accounting for the physical motion of the aircraft, a discrete dynamics associated with the flight plan, and a stochastic component given by the wind affecting the aircraft motion. An additional stochastic component (i.e., the radar measurement noise affecting the initial aircraft position) is present when predicting the aircraft future position. Here, we confine the description to the level flight case, with the aircraft flying at constant velocity (see [14], [25] for further extensions to the $3 \mathrm{D}$ case).

The continuous state components include the following variables: the aircraft positions $p=[x, y]$ in an inertial reference coordinate system with origin fixed at a point on the earth surface (i.e. the radar position), the heading angle $\psi$, and the aircraft mass $M$. The evolution of these variables is governed by the following equations:

$$
\left[\begin{array}{c}
\dot{x} \\
\dot{y} \\
\dot{\psi} \\
\dot{M}
\end{array}\right]=\left[\begin{array}{c}
V \cos (\psi) \cos (\gamma)+\nu_{x} \\
V \sin (\psi) \cos (\gamma)+\nu_{y} \\
\frac{1}{M} \frac{L \sin (\varphi)}{V \cos (\gamma)}-W_{c g f} \tan (\gamma) \\
-\eta T
\end{array}\right],
$$

where $V$ is the true air speed (assumed to be constant), $T$ is the engine thrust, $\eta$ represents the fuel consumption rate, $W_{c g f}$ is the cross track wind gradient factor and $L=\frac{C_{L} W_{s} \rho(z)}{2} V^{2}$ is the lift force, which depends on the air density $\rho(z)$ at the considered altitude $z$, the total wing surface $W_{s}$, and the lift coefficient $C_{L}$. Values for these parameters depend on the type of aircraft as detailed in the BADA document [11]. The stochastic wind $\nu(t, p)=\left[\nu_{x}, \nu_{y}\right]$ acts as a disturbance and enters additively the aircraft dynamics. The flight path angle $\gamma$ and the bank angle $\varphi$ are inputs set by the Flight Management System (FMS): when cruising at a constant altitude, $\gamma$ is set to zero so as to achieve a zero rate of climb/descent, whereas $\varphi$ is set based on the heading error and the cross track deviation from the nominal trajectory.

The discrete dynamics for level flight is derived from the flight plan and consists of the 2-D sequence of $s+1$ way-points $\left\{W P_{i}\right\}_{i=0}^{s}$ defining a piecewise linear nominal trajectory [26].

The stochastic wind component $\nu(t, p)$ is modeled as a Gaussian isotropic random field with zero mean and diagonal covariance matrix $C\left(t, p, t^{\prime}, p^{\prime}\right)=c\left(t, p, t^{\prime}, p^{\prime}\right) I_{2}$, where $I_{2}$ denotes the $2 \times 2$ identity matrix, $p, p^{\prime} \in \Re^{2}$ represent positions in the 2-D airspace and $t, t^{\prime} \in \Re$ time instants. Wind is correlated both in time and in space with the following correlation structure:

$$
c\left(t, p, t^{\prime}, p^{\prime}\right)=c_{z} c_{t}\left(\left|t-t^{\prime}\right|\right) c_{x y}\left(\left\|\begin{array}{l}
x-x^{\prime} \\
y-y^{\prime}
\end{array}\right\|\right),
$$

where $c_{z}$ is a suitable constant, whereas $c_{t}$ and $c_{x y}$ account for the temporal and spatial correlation, respectively, and are specified in [25] according to the empirical model in [32]. Note that the correlation structure is time-invariant since it depends on $t^{\prime}-t$ and not on $t$.

Additionally, since the aircraft position is tracked through some radar, a radar measurement noise is added to the initial aircraft position. Such a noise is modeled as an independent Gaussian process with zero mean and a diagonal covariance matrix $c_{r}^{2} I_{2}$, where $c_{r}$ is a suitable constant.

\section{REACH SET COMPUTATION}

Suppose that an aircraft is tracking some flight plan, and denote by $\bar{p}(t), t \in\left[t_{s}, t_{d}\right]$, its nominal position during the look-ahead time horizon $\left[t_{s}, t_{d}\right]$, where $t_{s}=0$ represents the current time instant. The aircraft future position is uncertain due to the stochastic wind affecting its motion and the radar measurement error on its initial position. We then denote by $p_{\delta}(t), t \in\left[t_{s}, t_{d}\right]$, the aircraft actual future position, where $\delta$ represents the stochastic uncertainty affecting the aircraft position and takes values in the set $\Delta$ of all possible realizations of the stochastic wind and the radar measurement noise. Our goal is determining the region of the airspace in the time cross space coordinates that will be occupied by the 
aircraft along the time horizon $\left[t_{s}, t_{d}\right]$ with probability at least $1-\epsilon$, where $\epsilon \in(0,1)$ is the violation parameter. This is the probabilistic reach set of level $1-\epsilon$.

\section{A. Reach set computation as a chance-constrained program}

Let us denote by $\mathcal{E}(\bar{p}, S)$ the ellipsoidal set centered at $\bar{p}$ with shape matrix $A=S^{T} S$, that is: $\mathcal{E}(\bar{p}, S):=\left\{p \in \Re^{2}\right.$ : $\left.\|S(p-\bar{p})\|^{2} \leq 1\right\}$. The region of the airspace that will be occupied by the aircraft during the time horizon $\left[t_{s}, t_{d}\right]$ is described as follows:

$$
\left\{\mathcal{E}\left(\bar{p}(t), S_{\theta}(t)\right): t \in\left[t_{s}, t_{d}\right]\right\},
$$

where $S_{\theta}(t), t \in\left[t_{s}, t_{d}\right]$, is a function of time finitely parameterized by some vector $\theta \in \Re^{d}$.

In order to determine the smallest region (1) containing all possible realizations of the aircraft trajectories except for a set of probability at most $\epsilon$, we formulate the following ChanceConstrained Optimization Program (C-COP):

$$
\begin{aligned}
& \underset{\theta \in \Re^{d}}{\operatorname{minimize}} \sum_{j=1}^{n_{s}} \log \operatorname{det} S_{\theta}\left(t_{j}\right)^{-1} \text { subject to: } \\
& S_{\theta}\left(t_{j}\right)=S_{\theta}\left(t_{j}\right)^{T}, j=1, \ldots, n_{s}, \\
& \mathcal{P}\left\{\delta: p_{\delta}\left(t_{j}\right) \in \mathcal{E}\left(\bar{p}\left(t_{j}\right), S_{\theta}\left(t_{j}\right)\right), j=1, \ldots, n_{s}\right\} \geq 1-\epsilon,
\end{aligned}
$$

where $\mathcal{P}$ is the probability distribution of the uncertainty parameter $\delta$ representing the stochastic wind and the radar measurement noise, and $\left\{t_{j}\right\}_{j=1}^{n_{s}}$ with $t_{s}=t_{1}<t_{2}<\cdots<$ $t_{n_{s}}=t_{d}$ are time instants obtained by uniformly gridding $\left[t_{s}, t_{d}\right]$

C-COPs like (2) are known to be difficult to solve and even NP-hard in some cases [30], [31]. One has then to head for an approximate solution. Here, we resort to a randomized method, called the scenario approach, as proposed earlier in [12]. The idea of the scenario approach is to replace the probabilistic constraint with a finite number $N$ of deterministic constraints, which are obtained by independently extracting $N$ "scenarios" $\delta^{(i)}, i=1, \ldots, N$, of the uncertainty $\delta$. The scenario version of problem (2) then becomes the following (convex) optimization program:

$$
\begin{aligned}
& \underset{\theta_{N} \in \Re^{d}}{\operatorname{minimize}} \sum_{j=1}^{n_{s}} \log \operatorname{det} S_{\theta_{N}}\left(t_{j}\right)^{-1} \text { subject to: } \\
& S_{\theta_{N}}\left(t_{j}\right)=S_{\theta_{N}}\left(t_{j}\right)^{T}, j=1, \ldots, n_{s}, \\
& p_{\delta^{(i)}}\left(t_{j}\right) \in \mathcal{E}\left(\bar{p}\left(t_{j}\right), S_{\theta_{N}}\left(t_{j}\right)\right), i=1, \ldots, N, j=1, \ldots, n_{s},
\end{aligned}
$$

which is always feasible since it consists in determining the minimum area set composed by $n_{s}$ ellipses, each one covering a finite number of points [4].

Let $\theta_{N}^{\star}$ be the solution to (3). Then, the following results holds (see [6] for a proof).

Theorem 1. Select a 'confidence parameter' $\beta \in(0,1)$. If $N$ is such that

$$
\sum_{i=0}^{d}\left(\begin{array}{c}
N \\
i
\end{array}\right) \epsilon^{i}(1-\epsilon)^{N-i} \leq \beta,
$$

where $d$ is the number of optimization variables and $\epsilon$ is the violation parameter, then, $\theta_{N}^{\star}$ satisfies

$$
\mathcal{P}\left\{\delta: p_{\delta}\left(t_{j}\right) \in \mathcal{E}\left(\bar{p}\left(t_{j}\right), S_{\theta_{N}^{\star}}\left(t_{j}\right)\right), j=1, \ldots, n_{s}\right\} \geq 1-\epsilon,
$$

with probability no smaller than $1-\beta$.

Remark 1. The explicit bound

$$
N \geq \frac{d+1+\ln (1 / \beta)+\sqrt{2(d+1) \ln (1 / \beta)}}{\epsilon}
$$

in [1] shows that the dependence of $N$ on the confidence parameter $\beta$ is logarithmic so that we can select $\beta$ to be such a small number as $10^{-10}$, in practice zero, and still $N$ does not grow significantly.

The reach sets obtained via the scenario program (3) are typically overapproximation of the actual reach sets, in that the scenario solution to a chance-constrained problem with an admissible violation $\epsilon$ typically results in an actual violation $\hat{\epsilon}$ that is much smaller than $\epsilon$ (see [7]). To overcome this issue and reduce the size of the reach sets while still guaranteeing that they contain all trajectories except for a set of probability smaller than or equal to $\epsilon$, we resort to the scenario approach with constraints removal introduced in [7]. The idea of this variant of the scenario approach for solving the chanceconstrained problem (2) is that of considering a finite number $N$ of possible scenarios $\delta^{(i)}, i=1, \ldots, N$, and remove a fraction $K=\lfloor\alpha N\rfloor, \alpha \in[0, \epsilon)$, of them so as to improve the cost function, i.e., reduce the area of the ellipsoidal reach sets. The resulting scenario program can be rewritten as follows:

$$
\begin{aligned}
& \underset{\theta_{N, K} \in \Re^{d}}{\operatorname{minimize}} \sum_{j=1}^{n_{s}} \log \operatorname{det} S_{\theta_{N, K}}\left(t_{j}\right)^{-1} \text { subject to: } \\
& S_{\theta_{N, K}}\left(t_{j}\right)=S_{\theta_{N, K}}\left(t_{j}\right)^{T}, j=1, \ldots, n_{s}, \\
& p_{\delta^{(i)}}\left(t_{j}\right) \in \mathcal{E}\left(\bar{p}\left(t_{j}\right), S_{\theta_{N, K}}\left(t_{j}\right)\right), \\
& i=1, \ldots, N \backslash\left\{i_{1}, \ldots, i_{K}\right\}, j=1, \ldots, n_{s}
\end{aligned}
$$

where $\left\{i_{1}, \ldots, i_{K}\right\} \subset\{1, \ldots, N\}$ are the indices of the constraints that are removed. If $N$ is appropriately chosen according to Theorem 2, then, the solution $\theta_{N, K}^{\star}$ to (5) is guaranteed to be chance-constrained feasible, with high confidence (see [7]).

Theorem 2. Select a 'confidence parameter' $\beta \in(0,1)$ and 'empirical violation parameter' $\alpha \in[0, \epsilon)$. If $N$ is such that

$$
\left(\begin{array}{c}
K+d \\
K
\end{array}\right) \sum_{i=0}^{K+d}\left(\begin{array}{c}
N \\
i
\end{array}\right) \epsilon^{i}(1-\epsilon)^{N-i} \leq \beta,
$$

( $d$ is the number of optimization variables and $K=\lfloor\alpha N\rfloor$ ), then, $\theta_{N, K}^{\star}$ satisfies

$$
\mathcal{P}\left\{\delta: p_{\delta}\left(t_{j}\right) \in \mathcal{E}\left(\bar{p}\left(t_{j}\right), S_{\theta_{N, K}^{\star}}\left(t_{j}\right)\right), j=1, \ldots, n_{s}\right\} \geq 1-\epsilon,
$$

with probability no smaller than $1-\beta$.

Remark 2. As for the choice of the empirical probability of violation $\alpha$, one should note that the closer $\alpha$ to the desired violation probability $\epsilon$ the better the approximation of the chance-constrained solution; yet, at the same time, it holds that $N \rightarrow \infty$ as $\alpha \rightarrow \epsilon$ (see [27] for an explicit bound on $N)$. Intuitively, if $\alpha$ equals $\epsilon$, then, $\mathcal{P}\left\{\delta: p_{\delta}\left(t_{j}\right) \in\right.$ 
$\left.\mathcal{E}\left(\bar{p}\left(t_{j}\right), S_{\theta_{N, K}^{\star}}\left(t_{j}\right)\right), j=1, \ldots, n_{s}\right\}$ will fluctuate around $1-\epsilon$ depending of the extracted samples of $\delta$, and it is not possible to guarantee that it is bigger than $1-\epsilon$ with high confidence for a finite $N$. Therefore, a proper value of $\alpha$ should be selected based on a trade-off between computational load and performance.

\section{B. Reach set computation for straight line flight plan}

Given that the computational effort involved in the reach set computation strongly depends on the number of optimization variables $d$, we adopt a 4-dimensional parametrization $\theta \in \Re^{4}$ for $S_{\theta}\left(t_{j}\right), j=1, \cdots, n_{s}$, so that the number of optimization variables is $d=4$ and the computational load is significantly reduced with respect to the fully parameterized case where all matrices $S_{\theta}\left(t_{j}\right), j=1, \cdots, n_{s}$, have free elements.

When the heading angle is $\psi=0^{\circ}, S_{\theta}\left(t_{j}\right), j=1, \cdots, n_{s}$, is parameterized as follows:

$$
S_{\theta}\left(t_{j}\right)=\left[\begin{array}{cc}
\theta_{1} \cdot j^{-1.3}+\theta_{2} & \theta_{3} \\
\theta_{3} & \theta_{4}
\end{array}\right],
$$

where $\theta=\left(\theta_{1}, \theta_{2}, \theta_{3}, \theta_{4}\right) \in \Re^{4}$. If we set $\theta_{3}=0$, the axes of the ellipses are parallel to the axes of the reference coordinate system. The shape matrix is then given by $A\left(t_{j}\right)=S_{\theta}\left(t_{j}\right)^{T} S_{\theta}\left(t_{j}\right)=\operatorname{diag}\left(\left(\theta_{1} \cdot j^{-1.3}+\theta_{2}\right)^{2}, \theta_{4}^{2}\right)$, and its eigenvalues are $\zeta_{1}\left(t_{j}\right)=\left(\theta_{1} \cdot j^{-1.3}+\theta_{2}\right)^{2}$ and $\zeta_{2}\left(t_{j}\right)=\theta_{4}^{2}$. Then, the semi-axis of length $\zeta_{1}\left(t_{j}\right)^{-1 / 2}=\left(\theta_{1} \cdot j^{-1.3}+\theta_{2}\right)^{-1}$ corresponds to the along-track error, whereas the semi-axis of length $\zeta_{2}\left(t_{j}\right)^{-1 / 2}=\theta_{4}^{-1}$ corresponds to the cross-track error. Note that $\zeta_{1}\left(t_{j}\right)^{-1 / 2}$ is growing as a function of time, which models the fact that the along-track error increases with time, whilst the cross-track error term is weakly dependent on time and hence it is modeled through a constant, see e.g. [29] and the references therein to justify this choice.

When $\psi \neq 0^{\circ}$, we can simply rotate clockwise both the nominal and the actual sampled trajectories $\bar{p}\left(t_{j}\right)$ and $p_{\delta}\left(t_{j}\right)$, $j=1, \cdots, n_{s}$, of an angle $\psi$ through the rotation matrix

$$
R_{\psi}=\left[\begin{array}{cc}
\cos \psi & \sin \psi \\
-\sin \psi & \cos \psi
\end{array}\right],
$$

so that we can still use the same parametrization of matrix $S_{\theta}\left(t_{j}\right)$ in (7) and just modify the optimization problem (3) by replacing $p_{\delta}\left(t_{j}\right)$ and $\bar{p}\left(t_{j}\right)$ with $R_{\psi} p_{\delta}\left(t_{j}\right)$ and $R_{\psi} \bar{p}\left(t_{j}\right)$, respectively.

If we aim at determining a shape matrix that applies to straight line legs associated with different heading angle and velocity pairs $\left(\psi_{k}, V_{k}\right), k=1, \ldots, m$, then, we have to solve the CCOP:

$$
\begin{aligned}
& \underset{\theta \in \Re^{d}}{\operatorname{minimize}} \sum_{j=1}^{n_{s}} \log \operatorname{det} S_{\theta}\left(t_{j}\right)^{-1} \text { subject to: } \\
& S_{\theta}\left(t_{j}\right)=S_{\theta}\left(t_{j}\right)^{T}, j=1, \ldots, n_{s} \\
& \mathcal{P}\left\{\delta:\left\|S_{\theta}\left(t_{j}\right) R_{\psi_{k}}\left(p_{k, \delta}\left(t_{j}\right)-\bar{p}_{k}\left(t_{j}\right)\right)\right\|^{2} \leq 1,\right. \\
& \left.j=1, \ldots, n_{s}, k=1, \ldots, m\right\} \geq 1-\epsilon,
\end{aligned}
$$

where $\bar{p}_{k}(t)$ denotes the nominal trajectory associated with the heading angle $\psi_{k}$ and the velocity $V_{k}$, whereas $p_{k, \delta}(t)$ is a possible realization of a trajectory associated with the same heading angle and velocity pair. The scenario approach can still be applied to find an approximate solution to (8), thus leading to the convex optimization program:

$$
\begin{aligned}
& \underset{\theta_{N} \in \Re^{d}}{\operatorname{minimize}} \sum_{j=1}^{n_{s}} \log \operatorname{det} S_{\theta_{N}}\left(t_{j}\right)^{-1} \text { subject to: } \\
& S_{\theta_{N}}\left(t_{j}\right)=S_{\theta_{N}}\left(t_{j}\right)^{T}, j=1, \ldots, n_{s}, \\
& \left\|S_{\theta_{N}}\left(t_{j}\right) R_{\psi_{k}}\left(p_{k, \delta^{(i)}}\left(t_{j}\right)-\bar{p}_{k}\left(t_{j}\right)\right)\right\|^{2} \leq 1, \\
& i=1, \ldots, N, j=1, \ldots, n_{s}, k=1, \ldots, m,
\end{aligned}
$$

to which the constraint removal procedure can be applied. In practice, this involves a few steps. First, the number of scenarios $N$ and the number of constraints to be removed $K$ are computed according to the bound (6) in Theorem 2. Then, $N$ scenarios $\delta^{(i)}, i=1, \ldots, N$, are extracted from $\Delta$ according to probability $\mathcal{P}$. The realizations of the actual aircraft trajectory associated with $\delta^{(i)}, i=1, \ldots, N$, are generated through the air traffic simulator for all pairs $\left\{\left(\psi_{k}, V_{k}\right)\right\}_{k=1}^{m}$. At each constraint removal iteration, a program like (9) but with a reduced number of constraints is solved via a standard convex optimization solver like that used in $c v x$ [15]. Solution $\theta_{h}^{\star}$ is computed for the current value of some index $h$, which is initially set equal to the number $K$ of constraints to be removed. Based on the computed ellipsoidal reach sets, all scenarios $\delta^{(i)}$ such that there is a trajectory realization $p_{k, \delta^{(i)}}$ that belongs to the boundary of the ellipsoidal reach sets at some time instant $t_{j}$ for some pair $\left(\psi_{k}, V_{k}\right)$, are determined. These are the scenarios to be removed. Then, $h$ is progressively reduced during the constraint removal iteration process. When $h=0$, the constraint removal procedure is completed. The corresponding solution $\theta_{0}^{\star}$ is the solution to the scenario program with constraint removal.

\section{PRoposed AIRCRAFT CD\&R SCHEME}

Let us consider $n$ aircraft flying in some region of the airspace. Each aircraft $i$ is following a straight line flight plan from a starting way-point denoted as $a_{i}$ to a destination way-point denoted as $b_{i}$, flying at some constant velocity $V_{i}$ during the finite time horizon $\left[t_{s}, t_{d}\right]$. Due to the uncertainty affecting its motion, the aircraft will not track exactly its nominal trajectory so that it might get close to some other aircraft, even if the nominal trajectories are separated. To account for this uncertainty, one can adopt the traditional minimum safety distance concept. This involves associating to each aircraft a "virtual circular reach set", which is a disk of radius equal to half the minimum safety distance centered in the aircraft nominal trajectory: a conflict occurs if at least two disks intersect at some time instant $t \in\left[t_{s}, t_{d}\right]$ [24].

Here, we adopt a different notion of conflict, which is based on the ellipsoidal reach sets associated with a certain probability level $1-\epsilon$ instead of the virtual circular reach sets. More precisely, a conflict is detected when the ellipsoidal reach sets of at least two aircraft intersect at some time instant, which from an implementation viewpoint can be verified via the numerical approach developed in [21]. Once a conflict is detected, it is resolved through the redesign of the aircraft flight plans. Since designing nominal trajectories such that the 
ellipsoidal reach sets do not intersect is not an easy task, we propose an iterative approach to tackle the problem.

Each iteration is based on a two-step procedure. In the first step, we adopt the approach proposed in [19] where circular sets with constant radius are used in place of ellipsoidal sets, which is easier to solve because the circle has rotational symmetry. Indeed, by using circular sets, conflict resolution is reformulated in [19] as a Second-Order Cone Program (SOCP). In the SOCP, flight plans are given by two-legged straight lines and the intermediate way-points are designed so as to minimize the traveled distance while avoiding disk intersections and accounting for constraints on the velocity and the turning angle at the same time (see [19]). In the second step, we perform conflict detection based on the flight plans designed in the first step and the actual ellipsoidal reach sets.

The overall iterative procedure is set up so as to look for the smallest radius to be used in the first step so as to obtain a conflict-free situation as outcome of the second step. A bisection algorithm is conceived to this purpose and the resulting CD\&R scheme is summarized in Algorithm 1.

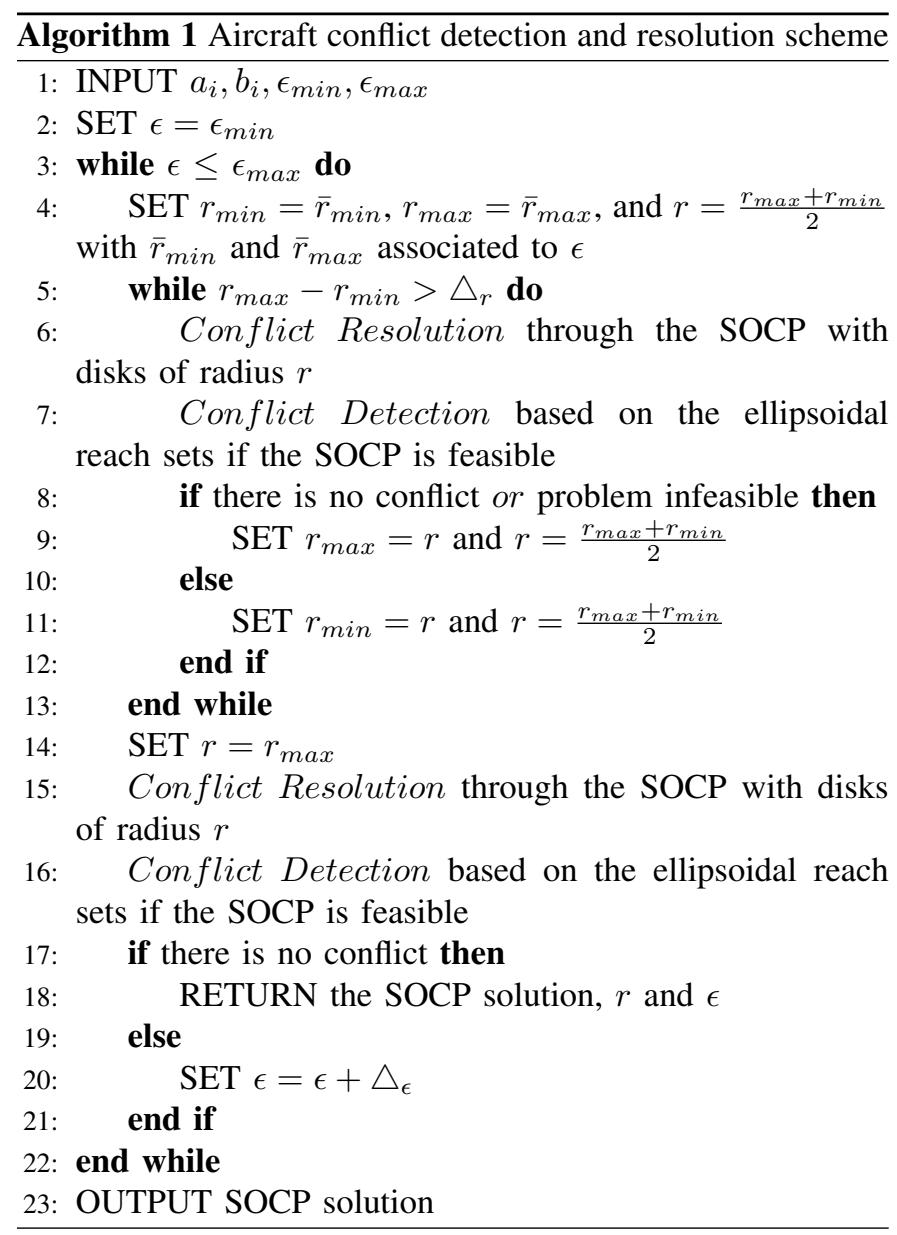

The bisection method is implemented in the inner while loop and the termination condition is $r_{\max }-r_{\min } \leq \triangle_{r}$, where $r_{\min }$ and $r_{\max }$ define the admissible range for radius $r$ and are reset at each iteration, according to the standard bisection method strategy. Precisely, at each iteration a certain radius $r$ is considered for conflict resolution. If either the resolution strategy is effective or it has no feasible solution, $r_{\max }$ is reduced to $\frac{r_{\min }+r_{\max }}{2}$. Otherwise, $r_{\min }$ is increased to $\frac{r_{\min }+r_{\max }}{2}$. Overall, the maximum number of iterations is approximately $\log _{2}\left(\frac{\bar{r}_{\max }-\bar{r}_{\min }}{\triangle_{r}}\right)$, where $\bar{r}_{\min }$ is the length of the minor semi-axis of the ellipsoidal reach set associated with the final time instant $t_{d}$ of the considered horizon $\left[t_{s}, t_{d}\right]$, whereas $\bar{r}_{\max }$ is the length of the major semi-axis of that same ellipsoidal set increased of a (small) quantity. This way if the radius $r$ were set equal to $\bar{r}_{\max }$, the conflict would be definitely solved because the circular reach sets approximations are all equal and large enough to include the ellipsoidal reach sets along the whole reference time horizon $\left[t_{s}, t_{d}\right]$. However, note that when $r$ becomes large, there may be no feasible solution to the SOCP because of some velocity and turning angle constraints embedded in it. Therefore, in Algorithm 1 an outer while loop is introduced where $\epsilon$ is increased so as to obtain smaller ellipsoidal reach sets and a feasible conflict-free solution. Meanwhile, the larger is $\epsilon$, the lower are the probabilistic guarantees on the safety of the resolution maneuver.

Remark 3 (design parameters). The key design parameter is $\epsilon$, setting the probability level for the probabilistic reach set and, hence, the level of risk taken when resolving conflicts on a mid-term time scale. Taking a large value for $\epsilon$ entails obtaining small reach sets and allowing the design of closer resolution trajectories for the aircraft flying in the same region of the airspace. This favors a better exploitation of the airspace and can be useful in case of high density traffic. On the contrary, taking a small value for $\epsilon$ means taking a lower level of risk and getting larger reach sets and resolution trajectories for the aircraft flying in the same region of the airspace that are far apart. As for $\alpha$, it defines the accuracy of the probabilistic reach sets computation via the scenario approach with constraints removal, and affects the off-line computational effort of the proposed CD\&R scheme. The value chosen for $\alpha$ is not much critical in that if it were even set to zero (no constraint removal), the actual risk would be smaller than $\epsilon$. The parameters involved in the online implementation of the conflict resolution scheme are the increments of the radius $r$ and of the level of risk $\epsilon$, and they should be set based on computational time considerations related to the specific set-up adopted in terms of computational resources.

\section{Numerical Results}

Numerical results in this section were obtained by using the Matlab version of the air traffic simulator described in [14] and [25], and available for download at http://people.epfl.ch/cgi-bin/people? id $=234671 \& o p=b i o \& l$ ang=en \& cvlang=en

\section{A. Reach set computation}

We next describe the results of the reach set computation that are obtained by generating multiple aircraft trajectory realizations given some predefined flight plan. In the experiments, we consider $m=9$ different heading angle and velocity pairs $\left(\psi_{k}, V_{k}\right), k=1, \ldots, m$, obtained combining 3 values for heading angle $\left(0^{\circ}, 120^{\circ}\right.$, and $\left.240^{\circ}\right)$ and velocity 
$(11.34 \mathrm{~km} / \mathrm{min}, 14.17 \mathrm{~km} / \mathrm{min}$, and $17.00 \mathrm{~km} / \mathrm{min})$. We refer to a 20 minutes time horizon $\left(\left[t_{s}, t_{d}\right]=[0,20]\right.$ minutes $)$, uniformly sampled every $\tau=0.5$ minute so as to obtain $\left\{t_{j}\right\}_{j=1}^{n_{s}}$ with $n_{s}=41.3$ different probability levels $1-\epsilon$ with $\epsilon=0.025,0.050,0.100$ are considered for reach set computation.

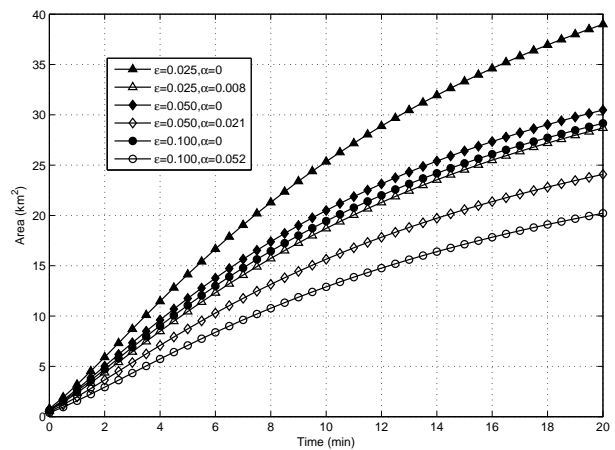

Fig. 1. Areas of the ellipsoidal reach sets computed through the proposed parameterized approach with ( $\alpha \neq 0$, lines with unfilled markers) and without ( $\alpha=0$, lines with filled markers) constraint removal as a function of time, for different $\epsilon$.

In Figure 1, the area of the ellipses as a function of time is depicted, in the case of no constraint removal (lines with filled markers) and constraint removal (lines with unfilled markers), for different values of $\epsilon$ and $\alpha$ when $\beta=10^{-8}$. The trend of area reduction is shown as $\epsilon$ grows. Table I reports the corresponding computation times when calculations are performed using Matlab R2015a, 64-bit, running on a personal computer with an Intel (R) Core (TM) i5-4590T CPU 2.00GHz processor, 4.00 GB RAM, and Windows 7 64-bit as operating system. Note that when no constraint removal is adopted $(\alpha=0)$ the CPU computation time decreases as $\epsilon$ grows, whereas this is not the case when $\alpha \neq 0$. More importantly, the CPU time increases significantly when $\alpha \neq 0$. This is not surprising given that constraints are progressively discarded through an iterative procedure, which involves repeatedly solving a (convex) optimization program. The required computation time could be lowered by adopting a more efficient coding on a platform with a higher performance. However, this is not much a concern here, since computation of the reach sets can be performed off-line.

TABLE I

CPU TIME $t_{C P U}$ FOR THE REACH SETS COMPUTATION

\begin{tabular}{ccccc}
\hline \hline$\epsilon$ & $\alpha$ & $N$ & $K$ & $t_{C P U}(\mathrm{~s})$ \\
\hline \multirow{2}{*}{0.025} & 0 & 1137 & - & 349.0 \\
& 0.008 & 3744 & 29 & 14736.0 \\
0.050 & 0 & 562 & - & 145.1 \\
& 0.021 & 2849 & 59 & 17183.5 \\
0.100 & 0 & 275 & - & 67.2 \\
& 0.052 & 2211 & 114 & 22627.9 \\
\hline \hline
\end{tabular}

\section{B. Aircraft $C D \& R$}

We now present some examples of aircraft encounters where some resolution action is needed, and discuss the influence of various factors on the corresponding resolution maneuver.
Each aircraft $i, i=1, \ldots, n$, is tracking some straight line nominal trajectory at constant velocity $V=14.17 \mathrm{~km} / \mathrm{min}$ along a 20 minutes time horizon $\left[t_{s}, t_{d}\right]=[0,20]$ minutes. Each aircraft velocity is constrained within the set $\left[V_{\min }, V_{\max }\right]$ where $V_{\min }=11.34 \mathrm{~km} / \mathrm{min}$ and $V_{\max }=17.00 \mathrm{~km} / \mathrm{min}$. The minimum turning angle is set equal to $90^{\circ}$. When resolving a conflict, the time associated with the intermediate way-point defining each aircraft nominal resolution maneuver is set equal to 10 minutes. As for the parameters $\triangle_{\epsilon}$ and $\triangle_{r}$ entering Algorithm 1, we set $\triangle_{\epsilon}=0.025$ and $\triangle_{r}=0.5 \mathrm{~km}$. The values of $\bar{r}_{\text {min }}$ and $\bar{r}_{\text {max }}$ corresponding to three values $0.025,0.050,0.100$ for $\epsilon$ are reported in Table II. Parameters $\bar{r}_{\text {min }}$ and $\bar{r}_{\text {max }}$ were computed based on the ellipsoidal reach sets obtained via the reach set computational approach with constraint removal (see Figure 1 for the adopted values of $\alpha$ ). $\bar{r}_{\max }$ is grown of $0.05 \mathrm{~km}$ with respect to the length of the major semi-axis of the ellipsoidal reach set at $t_{d}=20$ minutes.

TABLE II

THE ADMISSIBLE RANGE FOR RADIUS $r$ IN ALGORITHM 1

\begin{tabular}{cccc}
\hline \hline$\epsilon$ & 0.025 & 0.050 & 0.100 \\
$\bar{r}_{\min }$ & 0.653 & 0.595 & 0.525 \\
$\bar{r}_{\max }$ & 14.043 & 12.928 & 12.306 \\
\hline \hline
\end{tabular}

We consider conflict detection and resolution for multiaircraft encounters with a symmetric configurations. Aircraft starting way-points are symmetrically distributed on a circle of radius $141.7 \mathrm{~km}$ centered in $(148.160,148.160)$. Destination way-points are on the circle as well. Nominal trajectories pass through the center of the circle at time $t=10$ minutes in all considered encounters. Plots in the left column of Figure 2 show the original nominal trajectories when $n$ ranges from 3 to 8. Plots in the right column of Figure 2 show the corresponding redesigned conflict-free nominal trajectories for $\epsilon=0.025$. These resolution trajectories are optimal in that they adopt the minimum radius, called $r^{\star}$, when computing the intermediate waypoint. As $n$ grows from 3 to $8 r^{\star}$ is: $4.628,6.511,7.139$, 8.185, 8.394, and 7.557.

The time needed for multi-aircraft resolution is reported in Table III, which includes also the times involved in performing Conflict Detection (CD) and Conflict Resolution (CR) while running Algorithm 1. As for $\mathrm{CD}$, we used the Ellipsoidal toolbox [22] to check if two ellipses overlap when the distance between them was lower than the largest major axis. Note that the CD part is taking most of the total time. In our implementation, we check sequentially for pairwise overlaps. More efficient parallel implementations and a better performing platform could be adopted to reduce the computation time and allow for actual usage in tactical operations.

TABLE III

CPU TIME $t_{C P U}$ FOR THE MULTI-AIRCRAFT CD\&R ALGORITHM

\begin{tabular}{ccccccc}
\hline \hline$n$ & 3 & 4 & 5 & 6 & 7 & 8 \\
Total $t_{C P U}(\mathrm{~s})$ & 46.6 & 159.5 & 131.4 & 224.3 & 224.5 & 265.3 \\
$\mathrm{CD} t_{C P U}(\mathrm{~s})$ & 31.9 & 136.4 & 95.2 & 174.9 & 162.0 & 178.1 \\
$\mathrm{CR} t_{C P U}(\mathrm{~s})$ & 14.5 & 22.9 & 36.0 & 49.3 & 62.3 & 87.1 \\
\hline \hline
\end{tabular}

When $n$ is equal to 9 , there is no conflict-free resolution 


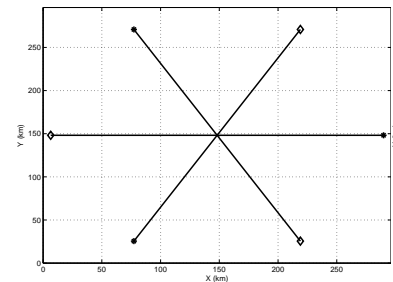

(a) 3 aircraft conflict

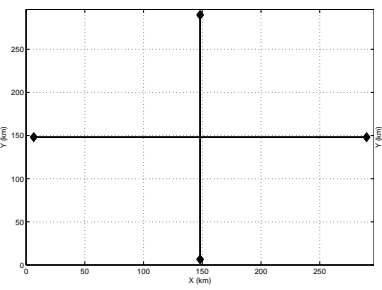

(c) 4 aircraft conflict

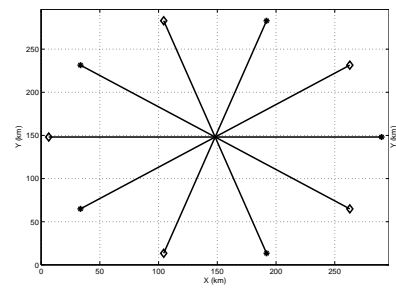

(e) 5 aircraft conflict

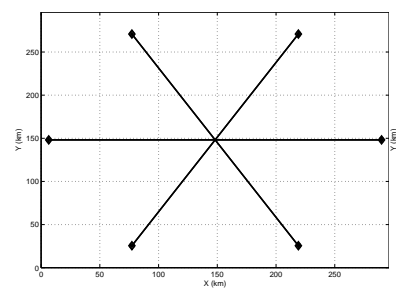

(g) 6 aircraft conflict

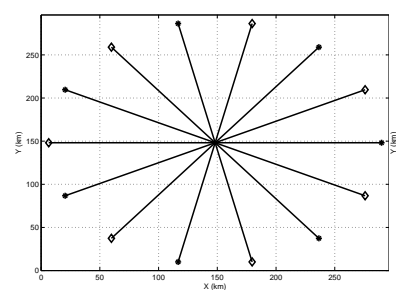

(i) 7 aircraft conflict

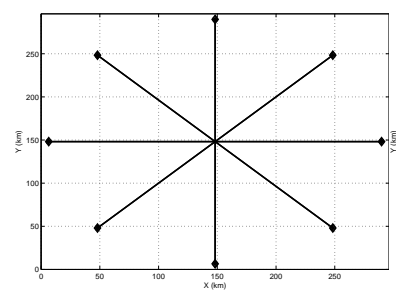

(k) 8 aircraft conflict

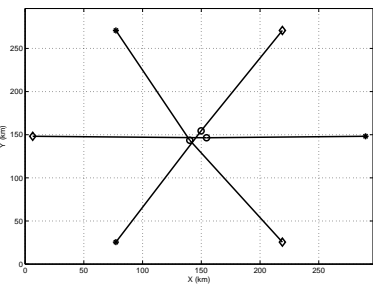

(b) 3 aircraft resolution

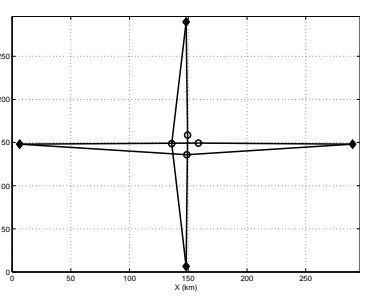

(d) 4 aircraft resolution

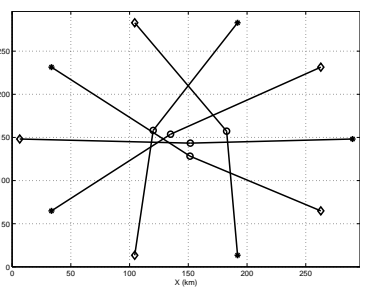

(f) 5 aircraft resolution

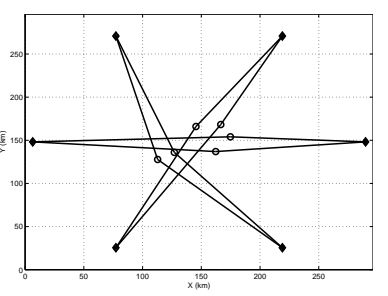

(h) 6 aircraft resolution

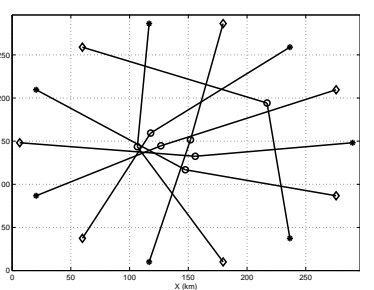

(j) 7 aircraft resolution

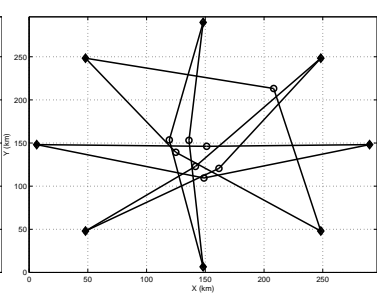

(1) 8 aircraft resolution
Fig. 2. Multi-aircraft encounters $(n=3,4,5,6,7,8)$. strategy for $\epsilon=0.025$, and not even for $\epsilon=0.050$ and $\epsilon=$ 0.100 . We can however get a feasible solution to the SOCP when we set $r$ equal to half the minimum safety distance (see Figure 3). This means that airspace area is densely packed, and aircraft should track accurately their nominal trajectories to avoid getting too close one to the other.

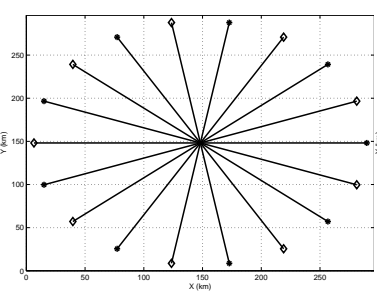

(a) 9 aircraft conflict

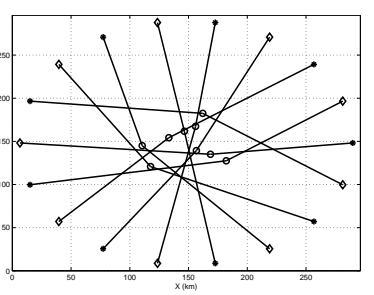

(b) 9 aircraft resolution
Fig. 3. 9 aircraft encounters and the conflict resolution maneuver guaranteeing the minimum nominal safety distance.

In order to investigate the influence of different values for $\epsilon$ on multi-aircraft conflict resolution, we focus on the symmetric encounter configuration with 6 aircraft and compute the resolution maneuver for $\epsilon=0.025, \epsilon=0.050$, and $\epsilon=0.100$. Figure 4 reports a time shot of the resolution strategy, where the ellipsoidal reach sets are plotted together with the disks of radius $r^{\star}$ at time 10 minutes for the three considered values of $\epsilon$. Not surprisingly, $r^{\star}$ is decreasing as a function of $\epsilon$ because the size of the ellipsoidal reach sets is decreasing with $\epsilon$. The values obtained for $r^{\star}$ are $8.185 \mathrm{~km}$ for $\epsilon=0.025,5.991 \mathrm{~km}$ for $\epsilon=0.050$, and $5.864 \mathrm{~km}$ for $\epsilon=0.100$, all values being larger than half the safety distance $9.26 \mathrm{~km}$ that is commonly used in avionics.

\section{CONCLUSions}

In this paper, we address multi-aircraft conflict detection and resolution through a stochastic reachability analysis approach, which rests on the computation of probabilistic reach sets to characterize the uncertainty on the aircraft future position, mainly due to wind. The main contribution of this work with respect to the existing literature consists of the development of a novel probabilistic conflict resolution scheme resting on reach sets computation that integrates in a stochastic setting the deterministic approach to conflict resolution proposed in [19]. As for the sources of uncertainty entering the stochastic model of the aircraft motion, it is worth pointing out that no Gaussianity or independence assumptions are needed in our approach. Moreover, although we refer to some specific stochastic model in this paper, the approach can be applied to more complex stochastic models, the only requirement being to have a simulator and to be able to generate multiple trajectories.

Regarding the contribution on the conflict resolution, admittedly, the adopted deterministic conflict resolution algorithm has some limitations since, for instance, intermediate waypoints of the resolution maneuvers of all aircraft are associated with the same nominal time. Future directions of research include overcoming such limitations so as to obtain a more flexible conflict detection and resolution scheme. 


\section{REFERENCES}

[1] T. Alamo, R. Tempo, and A. Luque. On the sample complexity of randomized approaches to the analysis and design under uncertainty. In American Control Conference (ACC), pages 4671-4676, Baltimore, MD, USA, June 2010.

[2] M. Althoff. Reachability analysis of nonlinear systems using conservative polynomialization and non-convex sets. In Hybrid Systems: Computation and Control, pages 173-182, 2013.

[3] M. Althoff, O. Stursberg, and M. Buss. Computing reachable sets of hybrid systems using a combination of zonotopes and polytopes. Nonlinear Analysis: Hybrid Systems, 4(2):233-249, 2010.

[4] S. Boyd and L. Vandenberghe. Convex Optimization. Cambridge University Press, 2009.

[5] G. Calafiore and M.C. Campi. Uncertain convex programs: Randomized solutions and confidence levels. Mathematical Programming, 102(1):2546, 2005.

[6] M.C. Campi and S. Garatti. The exact feasibility of randomized solutions of uncertain convex programs. SIAM Journal on Optimization, 19(3):1211-1230, 2008.

[7] M.C. Campi and S. Garatti. A sampling-and-discarding approach to chance-constrained optimization: Feasibility and optimality. Journal of Optimization Theory and Applications, 148(2):257-280, 2011.

[8] M.C. Campi, S. Garatti, and M. Prandini. The scenario approach for systems and control design. Annual Reviews in Control, 33(2):149-157, 2009.

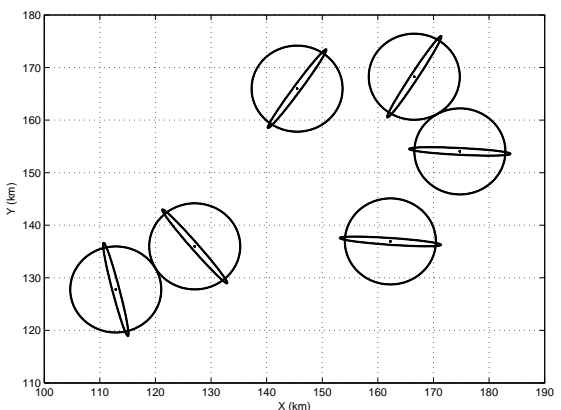

(a) $\epsilon=0.025$

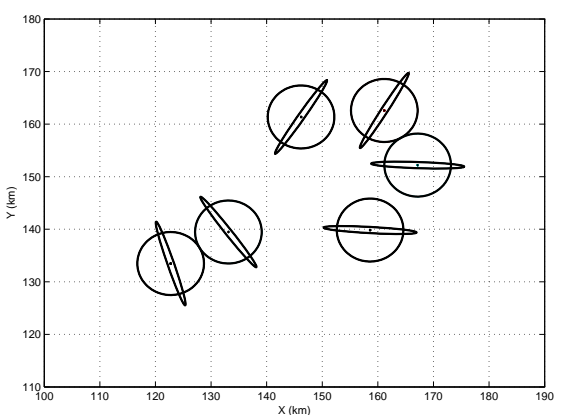

(b) $\epsilon=0.050$

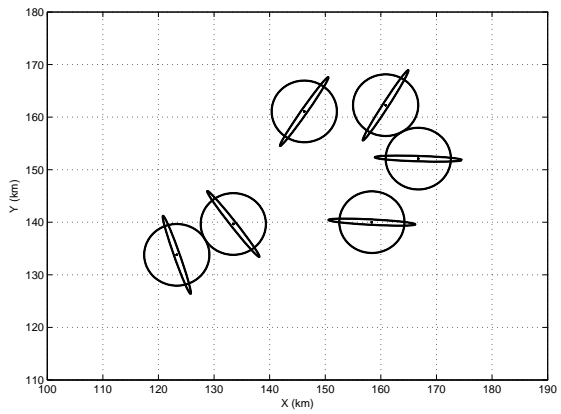

(c) $\epsilon=0.100$

Fig. 4. 6 aircraft resolution maneuvers at time 10 minutes for $\epsilon=0.025$, 0.050 , and 0.100 . Circles are the disks of radius $r^{\star}$ and ellipses are the actual reach sets. Nominal trajectories are marked with a $\bullet$.
[9] G. Chaloulos and J. Lygeros. Effect of wind correlation on aircraft conflict probability. Journal of Guidance Control and Dynamics, 30(6):1742, 2007

[10] H. Erzberger, R. Paielli, D. Isaacson, and M. Eshowx. Conflict detection and resolution in the presence of prediction error. In 1st USA/Europe Air Traffic Management Research Development Seminars, pages 50-56, Saclay, France, June 1997.

[11] EUROCONTROL. User Manual for the Base of Aircraft Data, 3.10 edition, 2012

[12] A. Falsone, F. Noce, and M. Prandini. A randomized approach to space debris footprint characterization. In 19th IFAC World Congress, Cape Town, South Africa, August 2014.

[13] G. Frehse, R. Kateja, and C. Le Guernic. Flowpipe approximation and clustering in space-time. In Hybrid Systems: Computation and Control, pages 203-212, 2013.

[14] W. Glover and J. Lygeros. A stochastic hybrid model for air traffic control simulation. Hybrid Systems:Computation and Control, Lecture Notes in Computer Science Volume 2993:372-386, 2004.

[15] M. Grant and S. Boyd. CVX: Matlab software for disciplined convex programming, version 2.0 beta. http://cvxr.com/cvx, 2013

[16] C. Le Guernic and A. Girard. Reachability analysis of linear systems using support functions. Nonlinear Analysis: Hybrid Systems, 4(2):250262, 2010.

[17] J. Hu, M. Prandini, and S. Sastry. Aircraft conflict prediction in the presence of a spatially correlated wind field. IEEE Transactions on Intelligent Transportation Systems, 6(3):326-340, 2005.

[18] I. Hwang and C.E. Seah. Intent-based probabilistic conflict detection for the next generation air transportation system. Proceedings of the IEEE, 96(12):2040-2059, 2008.

[19] M. Prandini J. Hu and S. Sastry. Optimal coordinated maneuvers for three dimensional aircraft conflict resolution. AIAA Journal of Guidance, Control and Dynamics, 25(5):888-900, 2002.

[20] J.K. Kuchar and L.C. Yang. A review of conflict detection and resolution modeling methods. IEEE Transactions on Intelligent Transportation Systems, 1(4):179-189, 2000

[21] A. Kurzhanski and P. Varaiya. Ellipsoidal techniques for reachability under state constraints. SIAM Journal on Control and Optimization, 45(4):1369-1394, 2006.

[22] A. A. Kurzhanskiy and P. Varaiya. Ellipsoidal toolbox. Technical Report UCB/EECS-2006-46, EECS Department, University of California, Berkeley, May 2006.

[23] A.A. Kurzhanskiy and P. Varaiya. Ellipsoidal techniques for reachability analysis of discrete-time linear systems. IEEE Transactions on Automatic Control, 52(1):26-38, Jan 2007.

[24] E.M. Feron L. Pallottino and A. Bicchi. Conflict resolution problems for air traffic management systems solved with mixed integer programming. IEEE Transactions on Intelligent Transportation Systems, 3(1):3-11, 2002.

[25] I. Lymperopoulos. Sequential Monte Carlo Methods in Air Traffic Management. Phd thesis, ETH, Department of Information Technology and Electrical Engineering, 2010.

[26] I. Lymperopoulos, A. Lecchini, W. Glover, J. M. Maciejowski, and J. Lygeros. A stochastic hybrid model for air traffic management processes. Technical report cued/f-infeng/tr.572, University of Cambridge, 2007.

[27] M. Prandini, S. Garatti, and R. Vignali. Performance assessment and design of abstracted models for stochastic hybrid systems through a randomized approach. Automatica, 50(11):2852-2860, 2014.

[28] M. Prandini and J. Hu. Application of reachability analysis for stochastic hybrid systems to aircraft conflict prediction. IEEE Transactions on Automatic Control, 54(4):913-917, 2009.

[29] M. Prandini, J. Hu, J. Lygeros, and S. Sastry. A probabilistic approach to aircraft conflict detection. IEEE Transactions on Intelligent Transportation Systems, 1(4):199-220, December 2000.

[30] A. Prèkopa. Stochastic Programming. Kluwer, Boston, MA, 1995.

[31] A. Prèkopa. Probabilistic programming. In A. Ruszczyǹski and A. Shapiro, editors, Stochastic Programming, volume 10 of Handbooks in Operations Research and Management Science, London, UK, 2003. Elsevier.

[32] S. Kim R.E. Cole, C. Richard and D. Bailey. An assessment of the $60 \mathrm{~km}$ Rapid Updata Cycle (RUC) with near real-time aircraft reports. Technical report, NASA, 1998.

[33] Y. Yang, J. Zhang, K. Cai, and M. Prandini. A stochastic reachability analysis approach to aircraft conflict detection and resolution. In 2014 IEEE Multi-conference on Systems and Control, Antibes, France, October 2014 